\title{
Disease Response Supplemental Qualifiers Dataset
}

National Cancer Institute

\section{Source}

National Cancer Institute. Disease Response Supplemental Qualifiers Dataset. NCI

Thesaurus. Code C147244.

A dataset containing supplemental information, specifically non-standard variables, to parent records in the disease response domain. 